\title{
Some remarks regarding the $(p, q)$-Fibonacci and Lucas octonion polynomials
}

\author{
Arzu Özkoç Öztürk ${ }^{\mathrm{a}^{*}}$ and Ayhan Porsuk ${ }^{\mathrm{a}}$ \\ ${ }^{a}$ Department of Mathematics, Faculty of Science and Arts, Düzce University, Düzce, Turkey \\ ${ }^{*}$ Corresponding author E-mail: arzuozkoc@duzce.edu.tr
}

\section{Article Info}

Keywords: Fibonacci Polynomials, Fibonacci Octonions, Generating Functions

2010 AMS: 05A15, 11B39, 11R52

Received: 30 January 2018

Accepted: 16 February 2018

Available online: 11 March 2018

\begin{abstract}
We investigate the $(p, q)$-Fibonacci and Lucas octonion polynomials. The main purpose of this paper is using of some properties of the $(p, q)$-Fibonacci and Lucas polynomials. Also for present some results involving these octonion polynomials, we obtain some interesting computational formulas.
\end{abstract}

\section{Introduction}

Fibonacci, Lucas, Pell and the other special numbers are the special case of the second order linear recurrence $R=\left\{R_{i}\right\}_{i=0}^{\infty}$ if the recurrence relation for $i \geq 2, R_{i}=P R_{i-1}-Q R_{i-2}$ holds for its terms, where $P$ and $Q$ are integers such that $D=P^{2}-4 Q \neq 0$ (to exclude a degenerate case) and $R_{0}, R_{1}$ are fixed integers. Define the sequences

$$
\begin{aligned}
& U_{n}=P U_{n-1}-Q U_{n-2} \\
& V_{n}=P V_{n-1}-Q V_{n-2}
\end{aligned}
$$

for $n \geq 2$. The characteristic equation of them is $x^{2}-P x+Q=0$ and hence the roots of it are $\alpha=\frac{P+\sqrt{D}}{2}$ and $\beta=\frac{P-\sqrt{D}}{2}$. So by Binet's formula, $U_{n}=\frac{\alpha^{n}-\beta^{n}}{\alpha-\beta}$ and $V_{n}=\alpha^{n}+\beta^{n}$. Further the generating function for $U_{n}$ and $V_{n}$ is

$$
\sum_{n=0}^{\infty} U_{n} x^{n}=\frac{x}{1-P x+Q x^{2}} \text { and } \sum_{n=0}^{\infty} V_{n} x^{n}=\frac{2-P x}{1-P x+Q x^{2}}
$$

$[8,9]$.

Polynomials can be defined by Fibonacci-like recursion relations are called Fibonacci polynomials. More mathematicians were involved in the study of Fibonacci polynomials. Let $p(x)$ and $q(x)$ be polynomials with real coefficients. The $(p, q)$-Fibonacci polynomials are defined by the recurrence relation

$$
F_{p, q, n+1}(x)=p(x) F_{p, q, n}(x)+q(x) F_{p, q, n-1}(x)
$$

with the initial conditions $F_{p, q, 0}(x)=0, F_{p, q, 1}(x)=1$. Also for the $p(x)$ and $q(x)$ polynomials with real coefficients the $(p, q)-$ Lucas polynomials are defined by the recurrence relation

$$
L_{p, q, n+1}(x)=p(x) L_{p, q, n}(x)+q(x) L_{p, q, n-1}(x)
$$

with the initial conditions $L_{p, q, 0}(x)=2, L_{p, q, 1}(x)=p(x)$. Let $\alpha_{1}(x)=\frac{p(x)+\sqrt{p^{2}(x)+4 q(x)}}{2}$ and $\alpha_{2}(x)=\frac{p(x)-\sqrt{p^{2}(x)+4 q(x)}}{2}$ denote the roots of the characteristic equation 


$$
\alpha^{2}-p(x) \alpha-q(x)=0
$$

on the recurrence relation of (1.2). Binet formulas for the $(p, q)$-Fibonacci polynomials and $(p, q)$-Lucas polynomials are

$$
F_{p, q, n}(x)=\frac{\alpha_{1}^{n}(x)-\alpha_{2}^{n}(x)}{\alpha_{1}(x)-\alpha_{2}(x)} \text { and } L_{p, q, n}(x)=\alpha_{1}^{n}(x)+\alpha_{2}^{n}(x)
$$

[10]

Note that

$$
\begin{aligned}
\alpha_{1}(x)+\alpha_{2}(x) & =p(x) \\
\alpha_{1}(x)-\alpha_{2}(x) & =\sqrt{p^{2}(x)+4 q(x)} \\
\alpha_{1}(x) . \alpha_{2}(x) & =-q(x) \\
\frac{\alpha_{1}(x)}{\alpha_{2}(x)} & =\frac{-\alpha_{1}^{2}(x)}{q(x)}, q(x) \neq 0 \\
\frac{\alpha_{2}(x)}{\alpha_{1}(x)} & =\frac{-\alpha_{2}^{2}(x)}{q(x)}, q(x) \neq 0 .
\end{aligned}
$$

In [5], they introduce $(p, q)$-Fibonacci quaternion polynomials that generalize $h(x)$-Fibonacci quaternion polynomials. Division algebras are defined on real numbers $\mathbb{R}$, complex numbers $\mathbb{C}$, quaternions $\mathbf{H}$, and octonions $\mathbb{Q}$. There are different types of sequences of quaternions like Fibonacci Quaternions, Split Fibonacci Quaternions and Complex Fibonacci Quaternions [1].

The octonions in Clifford algebra are a normed division algebra with eight dimensions over the real numbers larger than the quaternions. The field $\mathbb{Q} \cong \mathbb{C}^{4}$ of octonions

$$
\alpha=\sum_{s=0}^{7} \alpha_{s} e_{s}, \quad \alpha_{i} \in \mathbb{R}(i=0,1, \cdots, 7)
$$

is an eight-dimensional non-commutative and non-associative $\mathbb{R}$-field generated by eight base elements $e_{0}, e_{1}, \cdots, e_{6}$ and $e_{7}$ which satisfy the non-commutative and non-associative multiplication rules are listed in below Table.

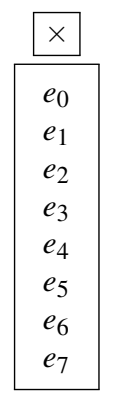

\begin{tabular}{|ccccccccc|}
\hline \multicolumn{1}{|c}{$e_{0}$} & $e_{1}$ & $e_{2}$ & $e_{3}$ & $e_{4}$ & $e_{5}$ & $e_{6}$ & $e_{7}$ \\
\hline$e_{0}$ & $e_{1}$ & $e_{2}$ & $e_{3}$ & $e_{4}$ & $e_{5}$ & $e_{6}$ & $e_{7}$ \\
$e_{1}$ & $-e_{0}$ & $e_{3}$ & $-e_{2}$ & $e_{5}$ & $-e_{4}$ & $-e_{7}$ & $e_{6}$ \\
$e_{2}$ & $-e_{3}$ & $-e_{0}$ & $e_{1}$ & $e_{6}$ & $e_{7}$ & $-e_{4}$ & $-e_{5}$ \\
$e_{3}$ & $e_{2}$ & $-e_{1}$ & $-e_{0}$ & $e_{7}$ & $-e_{6}$ & $e_{5}$ & $-e_{4}$ \\
$e_{4}$ & $-e_{5}$ & $-e_{6}$ & $-e_{7}$ & $-e_{0}$ & $e_{1}$ & $e_{2}$ & $e_{3}$ \\
$e_{5}$ & $e_{4}$ & $-e_{7}$ & $e_{6}$ & $-e_{1}$ & $-e_{0}$ & $-e_{3}$ & $e_{2}$ \\
$e_{6}$ & $e_{7}$ & $e_{4}$ & $-e_{5}$ & $-e_{2}$ & $e_{3}$ & $-e_{0}$ & $-e_{1}$ \\
$e_{7}$ & $-e_{6}$ & $e_{5}$ & $e_{4}$ & $-e_{3}$ & $-e_{2}$ & $e_{1}$ & $-e_{0}$ \\
\hline
\end{tabular}

The multiplication table for the basis of $\mathbb{Q}$

For $n \geq 0$, the Fibonacci octonion numbers that are given for the $n-t h$ classic Fibonacci $F_{n}$ number are defined by the following recurrence relations:

$$
\mathbb{Q}_{n}=\sum_{s=0}^{7} F_{n+s} e_{s} .
$$

Besides $h(x)$-Fibonacci octonion polynomials can be defined by [6] that generalized both Catalan's Fibonacci octonion polynomials $\Psi_{n}(x)$ and Byrd's Fibonacci octonion polynomials and also $k$ - Fibonacci octonion numbers. Moreover in [2] they derived the Binet formula and generating function of $h(x)$-Fibonacci octonion polynomial sequence.

Let $h(x)$ be a polynomial with real coefficients. The $h(x)$-Fibonacci octonion polynomials $\left\{O_{h, n}(x)\right\}_{n=0}^{\infty}$ are defined by the recurrence relation

$$
O_{h, n}(x)=\sum_{s=0}^{7} F_{h, n+s}(x) e_{s}
$$

where $F_{h, n}(x)$ is the $n-$ th $h(x)-$ Fibonacci polynomial in [2]. 


\section{Main theorems of the $(p, q)$-Fibonacci and Lucas octonion polynomials}

In the main section, we introduce the $(p, q)$-Fibonacci and Lucas octonion polynomials and formulate the Binet-style formula, the generating function and some identities of the $(p, q)$-Fibonacci octonion and Lucas octonion polynomial sequence. In [7], the authors obtained similar results for the $(p, q)$-Fibonacci and Lucas quaternion polynomials.

For $n \geq 0$ the Fibonacci octonion numbers that are given for the $n$-th classic Fibonacci $F_{n}$ number are defined in [4]. Also $(p, q)-$ Fibonacci octonions are investigated by [3].

So $(p, q)$-Fibonacci octonion polynomials $O F_{p, q, n}(x)$ are defined by the recurrence relation

$$
O F_{p, q, n}(x)=\sum_{k=0}^{7} F_{p, q, n+k}(x) e_{k}
$$

where $F_{p, q, n+k}(x)$ is the $(n+k)-t h(p, q)$-Fibonacci polynomial.

The initial conditions of this sequence are given by

$$
\begin{aligned}
O F_{p, q, 0}(x) & =\sum_{k=0}^{7} F_{p, q, k}(x) e_{k}=e_{1}+p(x) e_{2}+\left(p^{2}(x)+q(x)\right) e_{3}+\left(p^{3}(x)+2 p(x) q(x)\right) e_{4} \\
& +\left(p^{4}(x)+3 p^{2}(x) q(x)+q^{2}(x)\right) e_{5}+\left(p^{5}(x)+4 p^{3}(x) q(x)+3 p(x) q^{2}(x)\right) e_{6} \\
& +\left(p^{6}(x)+5 p^{4}(x) q(x)+6 p^{2}(x) q^{2}(x)+q^{3}(x)\right) e_{7}
\end{aligned}
$$

and

$$
\begin{aligned}
O F_{p, q, 1}(x) & =\sum_{k=0}^{7} F_{p, q, 1+k}(x) e_{k}=e_{0}+p(x) e_{1}+\left(p^{2}(x)+q(x)\right) e_{2}+\left(p^{3}(x)+2 p(x) q(x)\right) e_{3} \\
& +\left(p^{4}(x)+3 p^{2}(x) q(x)+q^{2}(x)\right) e_{4}+\left(p^{5}(x)+4 p^{3}(x) q(x)+3 p(x) q^{2}(x)\right) e_{5} \\
& +\left(p^{6}(x)+5 p^{4}(x) q(x)+6 p^{2}(x) q^{2}(x)+q^{3}(x)\right) e_{6} \\
& +\left(p^{7}(x)+6 p^{5}(x) q(x)+10 p^{3}(x) q^{2}(x)+4 p(x) q^{3}(x)\right) e_{7} .
\end{aligned}
$$

Also $O F_{p, q, n}(x)$ is written by a recurrence relation of order two;

$$
\begin{aligned}
O F_{p, q, n+1}(x) & =\sum_{k=0}^{7} F_{p, q, n+1+k}(x) e_{k} \\
& =\sum_{k=0}^{7}\left(p(x) F_{p, q, n+k}(x)+q(x) F_{p, q, n-1+k}(x)\right) e_{k} \\
& =p(x) \sum_{k=0}^{7} F_{p, q, n+k}(x) e_{k}+q(x) \sum_{k=0}^{7} F_{p, q, n-1+k}(x) e_{k}
\end{aligned}
$$

and thus,

$$
O F_{p, q, n+1}(x)=p(x) O F_{p, q, n}(x)+q(x) O F_{p, q, n-1}(x) .
$$

For the $n-t h(p, q)$-Lucas octonion polynomials $O L_{p, q, n}(x)=\sum_{k=0}^{7} L_{p, q, n+k}(x) e_{k}$, where $L_{p, q, n+k}$ is the $(n+k)-t h(p, q)-$ Lucas polynomial. For $n \geq 1$

$$
O L_{p, q, n+1}(x)=p(x) O L_{p, q, n}(x)+q(x) O L_{p, q, n-1}(x)
$$

with the initial conditions.

Theorem 2.1. The generating functions for the $(p, q)$-Fibonacci octonion polynomials $O F_{p, q, n}(x)$ and the $(p, q)-$ Lucas octonion polynomials $O L_{p, q, n}(x)$ are

$$
g_{O F}(t)=\frac{O F_{p, q, 0}(x)+\left[O F_{p, q, 1}(x)-p(x) O F_{p, q, 0}(x)\right] t}{1-p(x) t-q(x) t^{2}}
$$

and

$$
g_{O L}(t)=\frac{O L_{p, q, 0}(x)+\left[O L_{p, q, 1}(x)-p(x) O L_{p, q, 0}(x)\right] t}{1-p(x) t-q(x) t^{2}} .
$$

respectively. 
Proof. The generating function $g_{O F}(t)$ for $O F_{p, q, n}(x)$ is to be of the form

$$
\sum_{n=0}^{\infty} O F_{p, q, n}(x) t^{n}=O F_{p, q, 0}(x)+O F_{p, q, 1}(x) t+O F_{p, q, 2}(x) t^{2}+\cdots+O F_{p, q, n}(x) t^{n}+\cdots
$$

The formal power series expansions of $g_{O F}(t),-p(x) \operatorname{tg} O F(t)$ and $-q(x) t^{2} g_{O F}(t)$ are

$$
\begin{aligned}
g_{O F}(t) & =\sum_{n=0}^{\infty} O F_{p, q, n}(x) t^{n}=O F_{p, q, 0}(x)+O F_{p, q, 1}(x) t+O F_{p, q, 2}(x) t^{2} \\
& +\cdots+O F_{p, q, n}(x) t^{n}+\cdots \\
-p(x) t g_{O F}(t) & =-p(x) O F_{p, q, 0}(x) t-p(x) O F_{p, q, 1}(x) t^{2}-p(x) O F_{p, q, 2}(x) t^{3} \\
& -\cdots-p(x) O F_{p, q, n}(x) t^{n+1}-\cdots \\
-q(x) t^{2} g_{O F}(t) & =-q(x) O F_{p, q, 0}(x) t^{2}-q(x) O F_{p, q, 1}(x) t^{3}-q(x) O F_{p, q, 2}(x) t^{4} \\
& -\cdots-q(x) O F_{p, q, n}(x) t^{n+2}-\cdots
\end{aligned}
$$

respectively. So the expansion for $g_{O F}(t)-g_{O F}(t) p(x) t-g_{O F}(t) q(x) t^{2}$ is

$$
\begin{aligned}
g_{O F}(t)\left[1-p(x) t-q(x) t^{2}\right] & =O F_{p, q, 0}(x)+O F_{p, q, 1}(x) t-p(x) O F_{p, q, 0}(x) t \\
& +\left[O F_{p, q, 2}(x)-p(x) O F_{p, q, 1}(x)-q(x) O F_{p, q, 0}(x)\right] t^{2} \\
& +\left[O F_{p, q, 3}(x)-p(x) O F_{p, q, 2}(x)-q(x) O F_{p, q, 1}(x)\right] t^{3} \\
& +\ldots+\left[O F_{p, q, n}(x)-p(x) O F_{p, q, n-1}(x)-q(x) O F_{p, q, n-2}(x)\right] t^{n} \\
& +\ldots \\
& =O F_{p, q, 0}(x)+\left[O F_{p, q, 1}(x)-p(x) O F_{p, q, 0}(x)\right] t
\end{aligned}
$$

Hence $O F_{p, q, 0}(x)+\left[O F_{p, q, 1}(x)-p(x) O F_{p, q, 0}(x)\right] t$ is a finite series, so we can rewrite $\left[1-p(x) t-q(x) t^{2}\right] g_{O F}(t)=O F_{p, q, 0}(x)+\left[O F_{p, q, 1}(x)-\right.$ $\left.p(x) O F_{p, q, 0}(x)\right] t$ and hence

$$
g_{O F}(t)=\frac{O F_{p, q, 0}(x)+\left[O F_{p, q, 1}(x)-p(x) O F_{p, q, 0}(x)\right] t}{1-p(x) t-q(x) t^{2}}
$$

as we claimed.

Similarly, it can be also proved that $g_{O L}(t)=\frac{O L_{p, q, 0}(x)+\left[O L_{p, q, 1}(x)-p(x) O L_{p, q, 0}(x)\right] t}{1-p(x) t-q(x) t^{2}}$.

Lemma 2.2. For the generating function given in Theorem 2.1, we have

$$
\begin{aligned}
& g_{O F}(t)=\frac{1}{\alpha_{1}(x)-\alpha_{2}(x)}\left(\frac{O F_{p, q, 1}(x)-\alpha_{2}(x) O F_{p, q, 0}(x)}{1-\alpha_{1}(x) t}-\frac{O F_{p, q, 1}(x)-\alpha_{1}(x) O F_{p, q, 0}(x)}{1-\alpha_{2}(x) t}\right) \\
& g_{O L}(t)=\frac{1}{\alpha_{1}(x)-\alpha_{2}(x)}\left(\frac{O L_{p, q, 1}(x)-\alpha_{2}(x) O L_{p, q, 0}(x)}{1-\alpha_{1}(x) t}-\frac{O L_{p, q, 1}(x)-\alpha_{1}(x) O L_{p, q, 0}(x)}{1-\alpha_{2}(x) t}\right) .
\end{aligned}
$$

Proof. Using the expression of $g_{O F}(t)$ in Teorem 2.1 and (1.3), we found

$$
\begin{aligned}
& \frac{O F_{p, q, 0}(x)+\left[O F_{p, q, 1}(x)-p(x) O F_{p, q, 0}(x)\right] t}{1-p(x) t-q(x) t^{2}}=\frac{O F_{p, q, 0}(x)+\left[O F_{p, q, 1}(x)-p(x) O F_{p, q, 0}(x)\right] t}{\left(1-\alpha_{1}(x) t\right)\left(1-\alpha_{2}(x) t\right)} \\
= & \left(\frac{O F_{p, q, 0}(x)+\left[O F_{p, q, 1}(x)-\left(\alpha_{1}(x)+\alpha_{2}(x)\right) O F_{p, q, 0}(x)\right] t}{\left(1-\alpha_{1}(x) t\right)\left(1-\alpha_{2}(x) t\right)}\right) \times\left(\frac{\alpha_{1}(x)-\alpha_{2}(x)}{\alpha_{1}(x)-\alpha_{2}(x)}\right) \\
= & \frac{\left\{\begin{array}{c}
\alpha_{1}(x) O F_{p, q, 0}(x)+\alpha_{1}(x) O F_{p, q, 1}(x) t-\alpha_{1}^{2}(x) O F_{p, q, 0}(x) t \\
+\alpha_{1}(x) \alpha_{2}(x) O F_{p, q, 0}(x) t+\alpha_{2}^{2}(x) O F_{p, q, 0}(x) t+O F_{p, q, 1}(x)-O F_{p, q, 1}(x)
\end{array}\right)}{\left(\alpha_{1}(x)-\alpha_{2}(x)\right)\left(1-\alpha_{1}(x) t\right)\left(1-\alpha_{2}(x) t\right)} \\
= & \frac{\left\{\begin{array}{c}
O F_{p, q, 1}(x)\left(1-\alpha_{2}(x) t\right)+\alpha_{2}(x) O F_{p, q, 0}(x)\left(-1+\alpha_{2}(x) t\right) \\
+O F_{p, q, 1}(x)\left(-1+\alpha_{1}(x) t\right)+\alpha_{1}(x) O F_{p, q, 0}(x)\left(1-\alpha_{1}(x) t\right)
\end{array}\right\}}{\left(\alpha_{1}(x)-\alpha_{2}(x)\right)\left(1-\alpha_{1}(x) t\right)\left(1-\alpha_{2}(x) t\right)} \\
= & \frac{\left\{\begin{array}{c}
\left(1-\alpha_{2}(x) t\right)\left(O F_{p, q, 1}(x)-\alpha_{2}(x) O F_{p, q, 0}(x)\right) \\
-\left(1-\alpha_{1}(x) t\right)\left(O F_{p, q, 1}(x)-\alpha_{1}(x) O F_{p, q, 0}(x)\right)
\end{array}\right\}}{\left(\alpha_{1}(x)-\alpha_{2}(x)\right)\left(1-\alpha_{1}(x) t\right)\left(1-\alpha_{2}(x) t\right)} \\
= & \frac{1}{\alpha_{1}(x)-\alpha_{2}(x)}\left[\begin{array}{l}
O F_{p, q, 1}(x)-\alpha_{2}(x) O F_{p, q, 0}(x) \\
1-\alpha_{1}(x) t
\end{array}\right)
\end{aligned}
$$


Lemma 2.3. Let $F_{p, q, n}(x)$ and $L_{p, q, n}(x)$ be the $(p, q)$-Fibonacci and Lucas polynomials respectively. We have 1.

$$
\begin{aligned}
& F_{p, q, k+1}(x)-\alpha_{2}(x) F_{p, q, k}(x)=\alpha_{1}^{k}(x) \\
& F_{p, q, k+1}(x)-\alpha_{1}(x) F_{p, q, k}(x)=\alpha_{2}^{k}(x)
\end{aligned}
$$

2.

$$
\begin{aligned}
& \frac{L_{p, q, k+1}(x)-\alpha_{2}(x) L_{p, q, k}(x)}{\alpha_{1}(x)-\alpha_{2}(x)}=\alpha_{1}^{k}(x) \\
& \frac{\alpha_{1}(x) L_{p, q, k}(x)-L_{p, q, k+1}(x)}{\alpha_{1}(x)-\alpha_{2}(x)}=\alpha_{2}^{k}(x) .
\end{aligned}
$$

Proof. 1. We prove it by induction. Let $k=1$

$$
F_{p, q, 2}(x)-\alpha_{2}(x) F_{p, q, 1}(x)=p(x)-\alpha_{2}(x)=\alpha_{1}(x) .
$$

So the hypothesis is right for $k=1$. Let us assume that the equation is $F_{p, q, n}(x)-\alpha_{2}(x) F_{p, q, n-1}(x)=\alpha_{1}^{n-1}(x)$ for $k=n-1$. For $k=n$ it becomes

$$
\begin{aligned}
\alpha_{1}^{n}(x) & =\alpha_{1}^{n-1}(x) \alpha_{1}(x) \\
& =\left(F_{p, q, n}(x)-\alpha_{2}(x) F_{p, q, n-1}(x)\right) \alpha_{1}(x) \\
& =\alpha_{1}(x) F_{p, q, n}(x)-\alpha_{1}(x) \alpha_{2}(x) F_{p, q, n-1}(x) \\
& =\left(p(x)-\alpha_{2}(x)\right) F_{p, q, n}(x)-(-q(x)) F_{p, q, n-1}(x) \\
& =p(x) F_{p, q, n}(x)+q(x) F_{p, q, n-1}(x)-\alpha_{2}(x) F_{p, q, n}(x) \\
& =F_{p, q, n+1}(x)-\alpha_{2}(x) F_{p, q, n}(x) .
\end{aligned}
$$

So we get the desired result for the $(p, q)$-Fibonacci polynomials. 2. The $(p, q)$-Lucas polynomials can be proved similarly.

To derive the Binet Formulas for $O F_{p, q, n}(x)$ and $O L_{p, q, n}(x)$, we can give the following theorems.

Theorem 2.4. For $n \geq 0$, the Binet formula for the $(p, q)$-Fibonacci octonion polynomials $O F_{p, q, n}(x)$ and also $O L_{p, q, n}(x)$ is as follows

$$
\begin{gathered}
O F_{p, q, n}(x)=\frac{\alpha_{1}^{*}(x) \alpha_{1}^{n}(x)-\alpha_{2}^{*}(x) \alpha_{2}^{n}(x)}{\alpha_{1}(x)-\alpha_{2}(x)} \\
O L_{p, q, n}(x)=\alpha_{1}^{*}(x) \alpha_{1}^{n}(x)+\alpha_{2}^{*}(x) \alpha_{2}^{n}(x)
\end{gathered}
$$

where $\alpha_{1}^{*}(x)=\sum_{k=0}^{7} \alpha_{1}^{k}(x) e_{k}$ and $\alpha_{2}^{*}(x)=\sum_{k=0}^{7} \alpha_{2}^{k}(x) e_{k}$.

Proof. From Lemma 2.1, we get

$$
\begin{aligned}
& g_{O F}(t)=\frac{1}{\alpha_{1}(x)-\alpha_{2}(x)}\left[\left(O F_{p, q, 1}(x)-\alpha_{2}(x) O F_{p, q, 0}(x)\right)\right. \\
& \left.\sum_{n=0}^{\infty} \alpha_{1}^{n}(x) t^{n}-\left(O F_{p, q, 1}(x)-\alpha_{1}(x) O F_{p, q, 0}(x)\right) \sum_{n=0}^{\infty} \alpha_{2}^{n}(x) t^{n}\right] \\
& =\frac{1}{\alpha_{1}(x)-\alpha_{2}(x)}\left\{\begin{array}{c}
\sum_{k=0}^{7}\left(F_{p, q, 1+k}(x)-\alpha_{2}(x) F_{p, q, k}(x)\right) e_{k} \sum_{n=0}^{\infty} \alpha_{1}^{n}(x) t^{n} \\
-\sum_{k=0}^{\overline{7}}\left(F_{p, q, 1+k}(x)-\alpha_{1}(x) F_{p, q, k}(x)\right) e_{k} \sum_{n=0}^{\infty} \alpha_{1}^{n}(x) t^{n}
\end{array}\right\} \\
& =\frac{1}{\alpha_{1}(x)-\alpha_{2}(x)}\left[\sum_{k=0}^{7} \alpha_{1}^{k}(x) e_{k} \sum_{n=0}^{\infty} \alpha_{1}^{n}(x) t^{n}-\sum_{k=0}^{7} \alpha_{2}^{k}(x) e^{k} \sum_{n=0}^{\infty} \alpha_{2}^{n}(x) t^{n}\right] \\
& =\sum_{n=0}^{\infty} \frac{\alpha_{1}^{*}(x) \alpha_{1}^{n}(x)-\alpha_{2}^{*}(x) \alpha_{2}^{n}(x)}{\alpha_{1}(x)-\alpha_{2}(x)} t^{n} .
\end{aligned}
$$

Similarly, it can be also proved that $O L_{p, q, n}(x)=\alpha_{1}^{*}(x) \alpha_{1}^{n}(x)+\alpha_{2}^{*}(x) \alpha_{2}^{n}(x)$.

Theorem 2.5. (Catalan identity) Let the $(p, q)$-Fibonacci and Lucas octonion polynomials $O F_{p, q, n}(x)$ and $O L_{p, q, n}(x)$. For $n$ and $\alpha$, nonnegative integer numbers, such that $\alpha \leq n$, we have

$$
\begin{aligned}
& O F_{p, q, n+r}(x) O F_{p, q, n-r}(x)-O F_{p, q, n}^{2}(x)=\frac{(-1)^{r+n+1} \alpha_{1}^{*}(x) \alpha_{2}^{*}(x) q^{n-r}(x)\left(\alpha_{1}^{r}(x)-\alpha_{2}^{r}(x)\right)^{2}}{\left(\alpha_{1}(x)-\alpha_{2}(x)\right)^{2}} \\
& O L_{p, q, n+r}(x) O L_{p, q, n-r}(x)-O L_{p, q, n}^{2}(x)=(-1)^{r+n} \alpha_{1}^{*}(x) \alpha_{2}^{*}(x) q^{n-r}(x)\left(\alpha_{1}^{r}(x)-\alpha_{2}^{r}(x)\right)^{2}
\end{aligned}
$$


Proof. Using the identity (1.3), Lemma 2.2 and Theorem 2.2, we have

$$
\begin{aligned}
& O F_{p, q, n+r}(x) O F_{p, q, n-r}(x)-O F_{p, q, n}^{2}(x) \\
&=\left(\frac{\alpha_{1}^{*}(x) \alpha_{1}^{n+r}(x)-\alpha_{2}^{*}(x) \alpha_{2}^{n+r}(x)}{\alpha_{1}(x)-\alpha_{2}(x)}\right)\left(\frac{\alpha_{1}^{*}(x) \alpha_{1}^{n-r}(x)-\alpha_{2}^{*}(x) \alpha_{2}^{n-r}(x)}{\alpha_{1}(x)-\alpha_{2}(x)}\right) \\
&-\left(\frac{\alpha_{1}^{*}(x) \alpha_{1}^{n}(x)-\alpha_{2}^{*}(x) \alpha_{2}^{n}(x)}{\alpha_{1}(x)-\alpha_{2}(x)}\right)^{2} \\
&= \frac{\left\{\begin{array}{c}
-\alpha_{1}^{*}(x) \alpha_{2}^{*}(x) \alpha_{1}^{n-r}(x) \alpha_{2}^{n+r}(x) \\
-\alpha_{1}^{*}(x) \alpha_{2}^{*}(x) \alpha_{1}^{n+r}(x) \alpha_{2}^{n-r}(x) \\
+2 \alpha_{1}^{*}(x) \alpha_{2}^{*}(x) \alpha_{1}^{n}(x) \alpha_{2}^{n}(x)
\end{array}\right\}}{\left(\alpha_{1}(x)-\alpha_{2}(x)\right)^{2}} \\
&= \frac{-\alpha_{1}^{*}(x) \alpha_{2}^{*}(x) \alpha_{1}^{n}(x) \alpha_{2}^{n}(x)\left[\left(-\frac{\alpha_{2}^{2}(x)}{q(x)}\right)^{r}+\left(-\frac{\alpha_{1}^{2}(x)}{q(x)}\right)^{r}-2 \frac{\left(\alpha_{1}(x) \alpha_{2}(x)\right)^{r}}{q^{r}(x)}\right]}{\left(\alpha_{1}(x)-\alpha_{2}(x)\right)^{2}} \\
&= \frac{(-1)^{r+n+1} \alpha_{1}^{*}(x) \alpha_{2}^{*}(x) q^{n-r}(x)\left(\alpha_{1}^{r}(x)-\alpha_{2}^{r}(x)\right)^{2}}{\left(\alpha_{1}(x)-\alpha_{2}(x)\right)^{2}} .
\end{aligned}
$$

The other case can be proved similarly.

Theorem 2.6. (Cassini identity) For the $(p, q)$-Fibonacci octonion polynomials $O_{p, q, n}(x)$ and $(p, q)$-Lucas octonion polynomials $O L_{p, q, n}(x)$, we have

$$
\begin{aligned}
& O F_{p, q, n+1}(x) O F_{p, q, n-1}(x)-O F_{p, q, n}^{2}(x)=(-1)^{n} \alpha_{1}^{*}(x) \alpha_{2}^{*}(x) q^{n-1}(x) \\
& O L_{p, q, n+1}(x) O L_{p, q, n-1}(x)-O L_{p, q, n}^{2}(x)=(-1)^{1+n} \alpha_{1}^{*}(x) \alpha_{2}^{*}(x) q^{n-1}(x)\left(\alpha_{1}(x)-\alpha_{2}(x)\right)^{2}
\end{aligned}
$$

for any natural number $n$.

Theorem 2.7. Let $O_{p, q, n}(x)$ and $O L_{p, q, n}(x)$ be the $(p, q)$-Fibonacci and Lucas octonion polynomials respectively. Then for $n \geq 0$, we have

1.

$$
\begin{aligned}
& q(x)\left(O F_{p, q, n}(x)\right)^{2}+\left(O F_{p, q, n+1}(x)\right)^{2}=\frac{\left(\alpha_{1}^{*}\right)^{2}(x) \alpha_{1}^{2 n+1}(x)-\left(\alpha_{2}^{*}\right)^{2}(x) \alpha_{2}^{2 n+1}(x)}{\alpha_{1}(x)-\alpha_{2}(x)} \\
& q(x)\left(O L_{p, q, n}(x)\right)^{2}+\left(O L_{p, q, n+1}(x)\right)^{2}=\left(\alpha_{1}(x)-\alpha_{2}(x)\right)\left(\alpha_{1}^{*}\right)^{2}(x) \alpha_{1}^{2 n+1}(x)-\left(\alpha_{2}^{*}\right)^{2}(x) \alpha_{2}^{2 n+1}(x)
\end{aligned}
$$

2.

$$
\begin{aligned}
& O F_{p, q, 1}(x)-\alpha_{1}(x) Q F_{p, q, 0}(x)=\alpha_{2}^{*}(x) \\
& O F_{p, q, 1}(x)-\alpha_{2}(x) Q F_{p, q, 0}(x)=\alpha_{1}^{*}(x)
\end{aligned}
$$

and

$$
\begin{aligned}
& O L_{p, q, 1}(x)-\alpha_{1}(x) O L_{p, q, 0}(x)=\left(\alpha_{1}(x)-\alpha_{2}(x)\right) \alpha_{2}^{*}(x) \\
& O L_{p, q, 1}(x)-\alpha_{2}(x) O L_{p, q, 0}(x)=\left(\alpha_{1}(x)-\alpha_{2}(x)\right) \alpha_{1}^{*}(x) .
\end{aligned}
$$

Proof. Let us prove the identity 1.. From Theorem 2.2

$$
\begin{aligned}
q(x)\left(O F_{p, q, n}(x)\right)^{2}+\left(O F_{p, q, n+1}(x)\right)^{2} & =q(x)\left(\frac{\alpha_{1}^{*}(x) \alpha_{1}^{n}(x)-\alpha_{2}^{*}(x) \alpha_{2}^{n}(x)}{\alpha_{1}(x)-\alpha_{2}(x)}\right)^{2}+\left(\frac{\alpha_{1}^{*}(x) \alpha_{1}^{n+1}(x)-\alpha_{2}^{*}(x) \alpha_{2}^{n+1}(x)}{\alpha_{1}(x)-\alpha_{2}(x)}\right)^{2} \\
& =\frac{\left\{\begin{array}{c}
q(x)\left(\alpha_{1}^{*}\right)^{2}(x) \alpha_{1}^{2 n}(x)-2 q(x) \alpha_{1}^{*}(x) \alpha_{1}^{n}(x) \alpha_{2}^{*}(x) \alpha_{2}^{n}(x) \\
+q(x)\left(\alpha_{2}^{*}\right)^{2}(x) \alpha_{2}^{2 n}(x)+\left(\alpha_{1}^{*}\right)^{2}(x) \alpha_{1}^{2 n+2}(x) \\
-2 \alpha_{1}^{*}(x) \alpha_{1}^{n+1}(x) \alpha_{2}^{*}(x) \alpha_{2}^{n+1}(x)+\left(\alpha_{2}^{*}\right)^{2}(x) \alpha_{2}^{2 n+2}(x)
\end{array}\right\}}{\left(\alpha_{1}(x)-\alpha_{2}(x)\right)^{2}} \\
& =\frac{\left(\alpha_{1}^{*}\right)^{2}(x) \alpha_{1}^{2 n}(x)\left(q(x)-q(x) \frac{\alpha_{1}(x)}{\alpha_{2}(x)}\right)+\left(\alpha_{2}^{*}\right)^{2}(x) \alpha_{2}^{2 n}(x)\left(q(x)-q(x) \frac{\alpha_{2}(x)}{\alpha_{1}(x)}\right)}{\left(\alpha_{1}(x)-\alpha_{2}(x)\right)^{2}} \\
& =\frac{\left(\alpha_{1}^{*}\right)^{2}(x) \alpha_{1}^{2 n+1}(x)-\left(\alpha_{2}^{*}\right)^{2}(x) \alpha_{2}^{2 n+1}(x)}{\alpha_{1}(x)-\alpha_{2}(x)} .
\end{aligned}
$$

Also the proof of the identity 2 . is similar to 1 ..

Theorem 2.8. For the $(p, q)$-Fibonacci and Lucas octonion polynomials $O F_{p, q, n}(x)$ and $O L_{p, q, n}(x), n \geq 0$ we have following binomial sum formula for odd and even terms, 
1.

$$
\begin{gathered}
O F_{p, q, 2 n}(x)=\sum_{m=0}^{n}\left(\begin{array}{l}
n \\
m
\end{array}\right) q(x)^{n-m} p(x)^{m} O F_{p, q, m}(x) \\
O F_{p, q, 2 n+1}(x)=\sum_{m=0}^{n}\left(\begin{array}{l}
n \\
m
\end{array}\right) q(x)^{n-m} p(x)^{m} O F_{p, q, m+1}(x)
\end{gathered}
$$

2.

$$
\begin{gathered}
O L_{p, q, 2 n}(x)=\sum_{m=0}^{n}\left(\begin{array}{c}
n \\
m
\end{array}\right) q(x)^{n-m} p(x)^{m} O L_{p, q, m}(x) \\
O L_{p, q, 2 n+1}(x)=\sum_{m=0}^{n}\left(\begin{array}{c}
n \\
m
\end{array}\right) q(x)^{n-m} p(x)^{m} O L_{p, q, m+1}(x) .
\end{gathered}
$$

Proof. For 1. from (1.3) and Binet formulas, we get

$$
\begin{aligned}
& \sum_{m=0}^{n}\left(\begin{array}{c}
n \\
m
\end{array}\right) q(x)^{n-m} p(x)^{m} O F_{p, q, m}(x) \\
& =\sum_{m=0}^{n}\left(\begin{array}{c}
n \\
m
\end{array}\right) q(x)^{n-m} p(x)^{m} \frac{\alpha_{1}^{*}(x) \alpha_{1}^{m}(x)-\alpha_{2}^{*}(x) \alpha_{2}^{m}(x)}{\alpha_{1}(x)-\alpha_{2}(x)} \\
& =\frac{\alpha_{1}^{*}(x)}{\alpha_{1}(x)-\alpha_{2}(x)} \sum_{m=0}^{n}\left(\begin{array}{c}
n \\
m
\end{array}\right) q(x)^{n-m}\left(p(x) \alpha_{1}(x)\right)^{m} \\
& -\frac{\alpha_{2}^{*}(x)}{\alpha_{1}(x)-\alpha_{2}(x)} \sum_{m=0}^{n}\left(\begin{array}{c}
n \\
m
\end{array}\right) q(x)^{n-m}\left(p(x) \alpha_{2}(x)\right)^{m} \\
& =\frac{\alpha_{1}^{*}(x)}{\alpha_{1}(x)-\alpha_{2}(x)}\left(q(x)+p(x) \alpha_{1}(x)\right)^{n}-\frac{\alpha_{2}^{*}(x)}{\alpha_{1}(x)-\alpha_{2}(x)}\left(q(x)+p(x) \alpha_{2}(x)\right)^{n} \\
& =\frac{\alpha_{1}^{*}(x) \alpha_{1}^{2 n}(x)-\alpha_{2}^{*}(x) \alpha_{2}^{2 n}(x)}{\alpha_{1}(x)-\alpha_{2}(x)} \\
& =O F_{p, q, 2 n}(x) .
\end{aligned}
$$

Also the other cases for $O L_{p, q, n}(x)$ can be done similarly.

Theorem 2.9. The sums of the first $n$-terms of the sequences $O F_{p, q, n}(x)$ and $O L_{p, q, n}(x)$ are given by

$$
\sum_{m=0}^{n} O F_{p, q, m}(x)=\frac{-q(x) O F_{p, q, n}(x)-O F_{p, q, n+1}(x)+O F_{p, q, 0}(x)-\frac{\alpha_{1}^{*}(x) \alpha_{2}(x)-\alpha_{2}^{*}(x) \alpha_{1}(x)}{\alpha_{1}(x)-\alpha_{2}(x)}}{\left(\alpha_{1}(x)-1\right)\left(\alpha_{2}(x)-1\right)}
$$

and

$$
\sum_{m=0}^{n} O L_{p, q, m}(x)=\frac{-q(x) O L_{p, q, n}(x)-O L_{p, q, n+1}(x)+O L_{p, q, 0}(x)-\left[\alpha_{1}^{*}(x) \alpha_{2}(x)+\alpha_{2}^{*}(x) \alpha_{1}(x)\right]}{\left(\alpha_{1}(x)-1\right)\left(\alpha_{2}(x)-1\right)}
$$

respectively.

Proof. Using Binet formulas and the roots $\alpha_{1}(x), \alpha_{2}(x)$, we get

$$
\begin{aligned}
\sum_{m=0}^{n} O F_{p, q, m}(x) & =\frac{\alpha_{1}^{*}(x) \alpha_{1}^{m}(x)-\alpha_{2}^{*}(x) \alpha_{2}^{m}(x)}{\alpha_{1}(x)-\alpha_{2}(x)} \\
& =\frac{1}{\alpha_{1}(x)-\alpha_{2}(x)} \sum_{m=0}^{n}\left(\alpha_{1}^{*}(x) \alpha_{1}^{m}(x)-\alpha_{2}^{*}(x) \alpha_{2}^{m}(x)\right) \\
& =\frac{1}{\alpha_{1}(x)-\alpha_{2}(x)}\left(\alpha_{1}^{*}(x) \sum_{m=0}^{n} \alpha_{1}^{m}(x)-\alpha_{2}^{*}(x) \sum_{m=0}^{n} \alpha_{2}^{m}(x)\right) \\
& =\frac{1}{\alpha_{1}(x)-\alpha_{2}(x)}\left(\alpha_{1}^{*}(x) \frac{\alpha_{1}^{n+1}(x)-1}{\alpha_{1}(x)-1}-\alpha_{2}^{*}(x) \frac{\alpha_{2}^{n+1}(x)-1}{\alpha_{2}(x)-1}\right) \\
& =\frac{\alpha_{1}^{*}(x)\left(\alpha_{1}^{n+1}(x)-1\right)\left(\alpha_{2}(x)-1\right)-\alpha_{2}^{*}(x)\left(\alpha_{2}^{n+1}(x)-1\right)\left(\alpha_{1}(x)-1\right)}{\left(\alpha_{1}(x)-\alpha_{2}(x)\right)\left(\alpha_{1}(x)-1\right)\left(\alpha_{2}(x)-1\right)} \\
& =\frac{\left\{\begin{array}{c}
\left(\alpha_{1}^{*}(x) \alpha_{1}^{n+1}(x) \alpha_{2}(x)\right)-\left(\alpha_{1}^{*}(x) \alpha_{1}^{n+1}(x)\right)-\left(\alpha_{1}^{*}(x) \alpha_{2}(x)\right)+\alpha_{1}^{*}(x) \\
\left(\alpha_{2}^{*}(x) \alpha_{2}^{n+1}(x) \alpha_{1}(x)+\alpha_{2}^{*}(x) \alpha_{2}^{n+1}(x)+\alpha_{2}^{*}(x) \alpha_{2}(x)\right.
\end{array}\right\}}{\left(\alpha_{2}(x)\right)\left(\alpha_{1}(x)-1\right)\left(\alpha_{2}(x)-1\right)} \\
& =\frac{-q(x) O F_{p, q, n}(x)-O F_{p, q, n+1}(x)+O F_{p, q, 0}(x)-\frac{\alpha_{1}^{*}(x) \alpha_{2}(x)-\alpha_{2}^{*}(x) \alpha_{1}(x)}{\alpha_{1}(x)-\alpha_{2}(x)}}{\left(\alpha_{1}(x)-1\right)\left(\alpha_{2}(x)-1\right)} .
\end{aligned}
$$

The other cases for $O L_{p, q, n}(x)$ can be done similarly. 


\section{Conclusion}

Octonions have great importance as they are used in quantum physics, applied mathematics, graph theory. In this work, we introduce the $(p, q)$-Fibonacci and Lucas octonion polynomials and formulate the Binet-style formula, the generating function and some identities of the $(p, q)$-Fibonacci octonion and Lucas octonion polynomial sequence. Thus, in our future studies we plan to examine different quaternion and octonion polynomials and their key features.

\section{References}

[1] M. Akyigit, H. Kösal, M Tosun, Fibonacci Generalized Quaternions. Adv. Appl. Clifford Algebr. 24(3)(2014), 631-641.

[2] A. İpek, K Arı, On h(x)-Fibonacci Octonion Polynomials, Alabama Journal of Mathematics, 39, 2015.

[3] A. İpek, C. Çimen, On ( $p, q)$ Fibonacci Octonions. Mathematica Æterna, 6(6)(2016), 923-932.

[4] O. Keçilioğlu, İ. Akkus, The Fibonacci Octonions, Adv. App Clifford Algebras 25(2015) 151-158.

[5] G. Y. Lee and M. Asci, Some Properties of the $(p, q)$ - Fibonacci and $(p, q)$-Lucas Polynomials, Journal of Applied Mathematics, Volume 2012, Article ID 264842, 18 pages doi:10.1155/2012/264842.

[6] A .Nalli, P. Haukkanen, On generalized Fibonacci and Lucas Polynomials, Chaos Solitons and Fractals 42(2009) 3179-3186.

[7] A.Özkoç, A. Porsuk, A Note for the ( $p, q)$-Fibonacci and Lucas Quaternions Polynomials. Konuralp Journal of Mathematics. 5(2)(2017), 36-46.

[8] P. Ribenboim, My Numbers, My Friends, Popular Lectures on Number Theory, Springer-Verlag, New York, Inc. 2000.

[9] A Tekcan, A. Özkoç, M. Engür, M.E. Özbek, On Algebraic Identities on a New Integer Sequence with Four Parameters, Ars Combinatoria. 127(2016) 225-238.

[10] J. Wang, Some New Results for the (p,q)- Fibonacci and Lucas Polynomials. Advances in Difference Equations 2014, $2014: 64$. 